\title{
Prediction of Per Capita Ecological Carrying Capacity Based on ARIMA-LSTM in Tourism Ecological Footprint Big Data
}

\author{
Ping Xu \\ School of Humanities and Education, Xijing University, Xi'an 710123, Shaanxi, China \\ Correspondence should be addressed to Ping Xu; xuping@xijing.edu.cn
}

Received 29 December 2021; Revised 24 January 2022; Accepted 27 January 2022; Published 28 February 2022

Academic Editor: Zhongguo Yang

Copyright (C 2022 Ping Xu. This is an open access article distributed under the Creative Commons Attribution License, which permits unrestricted use, distribution, and reproduction in any medium, provided the original work is properly cited.

\begin{abstract}
Reasonable and effective regional ecological evaluation and analysis methods can be an effective help for urban sustainable development, but there are still some errors in the current ecological prediction and analysis methods. To solve this problem, this paper proposes a prediction method of per capita ecological carrying capacity based on the autoregressive integrated moving average model (ARIMA) and long short-term memory (LSTM). First, the method improves the ecological footprint model based on energy analysis and constructs a comprehensive regional ecological data model; considering the complex characteristics of ecological data set, based on the ARIMA network model and LSTM model, a reliable and efficient big data prediction model of per capita ecological carrying capacity is established by analyzing the linear or nonlinear data sets in the data set. Finally, according to the actual ecological data set collected in Shenzhen, China, the results show that the economic and ecological trend of Shenzhen is generally good.
\end{abstract}

\section{Introduction}

With the rapid growth of population, the natural resources, environment, and economy are becoming increasingly serious, which leads to major problems such as resource depletion, environmental degradation, and ecological damage $[1,2]$. Human activities have more and more influence on the Earth ecosystem. To correctly evaluate the pressure of human activities on the regional natural environment and the carrying capacity of the regional ecosystem $[3,4]$, and to analyze the relationship between human and nature, environment and economy are important basic problems to realize the sustainable development of social economy.

Before making a regional analysis, we need to establish a certain evaluation index system, while the traditional evaluation methods of ecological capacity state-space and comprehensive evaluation have some limitations [5-7]. The state-space method is an effective method to quantitatively describe the state of the system in the three-dimensional Euclidean space, but the state-space method can only judge whether the study area is overloaded and cannot give the specific ecological carrying capacity [8]. The comprehensive evaluation method is vulnerable to human subjective factors when constructing a certain evaluation index system [9].

With the continuous innovation of evaluation methods and means, ecological footprint analysis as a quantitative analysis method has attracted people's attention [10]. The ecological footprint analysis method was put forward by the ecological economist Rees in 1992. This method is an important tool to measure the utilization of natural resources and sustainable development. However, it should be noted that in the regional scale ecological sustainability assessment, only based on the ecological surplus or deficit obtained by comparing the ecological footprint and ecological carrying capacity within the region to determine the regional sustainability will produce large prediction error [11].

Thanks to the development of big data technology and Internet of things technology, the intelligent algorithm provides a new solution for regional ecological analysis $[12,13]$. In Reference [14], the generalized regression neural network was used to build the ecological prediction and evaluation model to realize the analysis and evaluation of Jinan water area; In Reference [15], a new model with high 
prediction accuracy based on support vector machine is proposed to analyze the sustainable development of Beijing. In Reference [16], an artificial bee colony algorithm is used to optimize the radial basis function neural network, and a new prediction model of urban ecological carrying capacity is constructed, which provides a certain theoretical basis for the government's sustainable development decision. However, the above methods ignore the complexity of the ecological data itself, and the regional ecological data have linear and nonlinear attributes [17]. If only the single characteristic of the data is discussed, it is difficult to achieve accurate and effective data feature extraction, which will easily lead to certain errors in the model.

In view of the problems existing in the current analysis methods, in order to more accurately analyze the regional per capita ecological carrying capacity, this paper proposes a new ecological footprint analysis method based on the big data technology.

This paper starts from the system theory method, uses the energy analysis method to improve the ecological footprint model, and establishes a more comprehensive ecological footprint data set. In view of the complex characteristics of big data of ecological footprint, this paper improves the long short-term memory (LSTM) prediction network based on the ARIMA and comprehensively analyzes the linear and nonlinear characteristics of ecological data to achieve the efficient prediction of the ecological capacity in the study area.

The main contents of the remaining chapters are as follows: Section 2 describes the ecological data model and research area; Section 3 introduces the prediction model of per capita ecological carrying capacity based on ARIMALSTM combination; Section 4 is based on the actual data collected in the Shenzhen area to achieve simulation verification; Section 5 is the conclusion and prospect of this paper.

\section{Research Areas and Methods}

\subsection{Research Methods}

2.1.1. Ecological Footprint Method. The ecological footprint method is mainly to convert regional resources and energy consumption into the land area needed for the production of these materials. The calculation in this paper compares the ecological footprint demand and ecological carrying capacity. If the ecological footprint demand exceeds the ecological carrying capacity of the region, it is an ecological deficit, which means that the regional land production pressure load is large and the ecosystem is unstable; on the contrary, if the ecological footprint demand is less than the regional ecological carrying capacity, it is ecological surplus, indicating that the regional land use is reasonable and the land resources can be used sustainably [18]. The calculation formula of per capita ecological footprint is as follows:

$$
\text { ef }=\sum_{j}^{6}\left(\sum \frac{C_{i}}{P_{i} \times N} \times E_{j}\right),
$$

where $i$ is the type of consumer goods and inputs; $j$ is the type of land use; $C_{i}$ is the annual consumption of consumer goods $i$; $P_{i}$ is the global average annual production capacity of the $i$ consumer goods; $E_{j}$ is the equilibrium factor of $j$ land; $N$ is the number of people participating in consumption; and ef is the per capita ecological footprint. The calculation formula of per capita ecological carrying capacity is as follows:

$$
e c=\sum_{j}^{6}\left(\frac{A_{j}}{N} \times Y_{j} \times E_{j}\right),
$$

where $j$ is the type of land use; $A_{j}$ is the area of a certain kind of bioproductive land in a country or region; $Y_{j}$ is yield factor; $E_{j}$ is the equilibrium factor of the $j$ land; $N$ is the population; and $e c$ is the per capita available ecosystem carrying capacity.

According to the actual production and consumption situation of specific cities, the indicators selected for the establishment of ecological footprint account are rice, wheat, rapeseed, vegetables, pork, eggs, fruits, beef and mutton, milk, aquatic products, coke, gasoline, diesel, liquefied gas, natural gas, and electricity. In addition, in order to make the calculation results more accurate and reduce errors, combined with the survey and evaluation results of agricultural land comprehensive production capacity in Guangdong Province, the equilibrium factors of cultivated land, grassland, forest land, water area, construction land, and fossil fuel in Shenzhen City were determined as 2.12, 1.71, 0.69, $0.41,1.46$, and 2.87 , respectively, and the corresponding yield factors were $1.35,1.01,0.41,1.31,1.28$, and 1.48, respectively.

2.1.2. Open System Ecological Footprint Theory Based on Energy Analysis. It should be noted that the aforementioned traditional ecological footprint model has limitations for the rating of regional development. In the regional scale ecological sustainability assessment, it will produce large errors when judging the regional sustainability only based on the ecological surplus or deficit obtained by comparing the ecological footprint within the region with the ecological carrying capacity [19].

The energy method starts from the system theory, based on the advantages of flow calculation, subdivision of energy conversion rate, and integration of human labor into the system evaluation, to make up for the shortcomings of the traditional ecological footprint model. Therefore, based on the purpose of expanding supply and redefining ecological carrying capacity, this article further improves the traditional ecological footprint model on the basis of previous research, fully considers the contribution of human beings, and incorporates import and export into the evaluation framework.

The calculation steps and methods of the improved ecological footprint model are as follows.

Step 1. Establishing the exchange of energy and ecological footprint.

The input of global renewable resources is the basis of sustainable development and also the source of ecological 
carrying capacity. Therefore, the global annual natural energy input corresponds to the global annual ecological carrying capacity.

The calculation formula of energy density per hectare $P$ is as follows (unit sej/ghm ${ }^{2}$ ):

$$
P=\frac{G E I}{G E C}
$$

where GEI is all natural energy input of the world in one year and GEC is the global ecological carrying capacity in a year.

Step 2. Calculating ecological footprint.

First, the consumption items are divided, and then, the solar energy value of each consumption item in the region is calculated based on the energy conversion rate, and finally, the per capita solar energy value of each consumption item is calculated. Then, the per capita energy of each consumption item is converted into the corresponding bioproductive land area, that is, the ecological carrying area of consumption. Then, the calculation formula of ecological footprint is rewritten as follows:

$$
\text { ef }=\sum_{i=1}^{n} a_{i}=\sum_{i=1}^{n} \frac{\left(x_{i} \times T_{i}\right)}{P},
$$

where $i$ is the resource type; $a_{i}$ is the per capita ecological footprint of resource $i ; x_{i}$ is the per capita consumption of resource $i$; $T_{i}$ is the energy conversion rate of the $i$ resource; and $P$ is the energy density per hectare.

Step 3. Calculation of ecological carrying capacity.

The total potential ecological carrying capacity corresponding to the input of all renewable natural energy values of a system is defined as the local maximum potential ecological carrying capacity, which represents the maximum ecological carrying capacity when the system potential is fully exploited and utilized in an ideal state $[20,21]$. The calculation formula of ecological carrying capacity is rewritten as follows:

$$
e c=\sum_{i=1}^{n}\left(r_{i} \times T_{i}\right) / P,
$$

where $n$ is the input type of local renewable natural energy; $r_{i}$ is the original value of the $i$ natural energy value; $T_{i}$ is the energy conversion rate of resource $i$. The energy contained in all natural resources comes from solar energy. In order to avoid repeated calculation, the corresponding bioproductive land area converted from the maximum per capita energy value of renewable resources is taken as the per capita ecological carrying capacity value of the study area.

The output ecological carrying capacity of local products, that is, the corresponding ecological carrying capacity of local actually produced products, is as follows. The calculation formula is similar to the calculation formula of ecological footprint, including three main production projects: biological resource output, energy resource output, and primary industrial product output.

$$
\text { oec }=\sum_{i=1}^{n} c_{i}=\sum_{i=1}^{n} \frac{\left(y_{i} \times T_{i}\right)}{P},
$$

where oec is the carrying capacity of per capita product output; $c_{i}$ is the per capita ecological carrying capacity of product $i$; and $y_{i}$ is the per capita production of resource $i$.

Step 4. Calculating local labor services and the corresponding ecological carrying capacity of imports and exports.

Pay attention to the role of human beings, and measure the contribution of human beings in the ecological economic system by the income of regional population labor services. The service income multiplied by the global energy/currency ratio is converted into energy and then converted into the corresponding ecological carrying capacity. The global energy/currency ratio is equal to the total global energy consumption divided by the global GDP of the current year. The total global annual energy consumption includes renewable resource energy consumption and nonrenewable resource energy consumption.

The circulation of ecological carrying capacity is realized through the import and export of regional products. In view of the incomplete statistics of product import and export, the total amount of regional import and export currency in the current year is multiplied by the global energy/currency ratio and then converted into ecological carrying capacity as a substitute. The calculation formula is as follows:

$$
\text { MEC }=\frac{M_{p}}{P_{e / m}},
$$

where MEC is the per capita monetary carrying capacity represented by per capita labor income or import and export value; $M_{p}$ is the per capita labor income or per capita import and export currency amount; and $P_{e / m}$ is the energy/currency ratio.

Step 5. Sustainable development degree of the comprehensive evaluation system.

This paper calculates the ecological deficit or surplus and comprehensively analyzes the role of regional labor ecological carrying capacity and carrying capacity circulation in regional development, so as to measure the sustainable development of the study area.

2.1.3. Diversity of Ecological Footprint and Calculation of Economic Development Ability. The area of different land types is an important index for measuring the diversity of the eco-economic system [22]. Shannon Weaver formula was used to calculate the diversity index of ecological footprint, and Ulanowicz formula was used to calculate the development capacity of the ecological economic system.

$$
\begin{aligned}
H & =-\sum p_{i} \ln p_{i} \quad(i=1,2, \ldots), \\
C & =e f *\left[-\sum p_{i} \ln p_{i}\right]=\text { ef } * H \quad(i=1,2, \ldots),
\end{aligned}
$$


where $H$ is the diversity index of ecological footprint; $p_{i}$ is the proportion of type $i$ land footprint in the total ecological footprint; and $C$ is development capacity. The closer the distribution of ecological footprint is to equality, the higher the diversity is.

2.2. Study Area. As shown in Figure 1, this paper chooses Shenzhen, Guangdong Province as the area for analysis and research in this paper. Its land area is $113^{\circ} 46^{\prime} \sim 114^{\circ} 37^{\prime}$ east longitude and $22^{\circ} 27^{\prime} \sim 22^{\circ} 52^{\prime}$ north latitude, with a total area of $1952.84 \mathrm{~km}^{2}$. Shenzhen is adjacent to Hong Kong, Dongguan, and Huizhou. The terrain is high in the southeast and low in the northwest. The coastal area in the west is the coastal plain. The annual average rainfall is $1933.3 \mathrm{~m}^{2}$, and the sunshine is 2120.5 hours. The coastline of Shenzhen is $230 \mathrm{~km}$ long, with abundant marine resources, excellent bay ports, and superior access to the sea. Shenzhen currently governs 6 county-level organizational districts, namely, Luohu, Futian, Nanshan, and Yantian within the special zone and Baoan and Longgang outside the special zone.

\section{Prediction of Per Capita Ecological Carrying Capacity Based on the ARIMA-LSTM Combined Model}

3.1. Introduction of the ARIMA Model. ARIMA model mainly deals with the random nonstationary time series [23]. It combines the moving average model and the autoregressive model to make a difference transformation to predict the future trend [24].

The expression of $\mathrm{AR}$ is as follows:

$$
y_{t}=\Phi_{1} y_{t-1}+\Phi_{2} y_{t-2}+\cdots+\Phi_{p} y_{t-p}+\varepsilon_{t}
$$

where $p$ is the order of the model; $y_{t}$ represents the current forecast data; $y_{t-1}, y_{t-2}, \ldots$, and $y_{t-p}$ represent the historical observation values at different times; $\Phi_{1}, \Phi_{2}, \ldots$, and $\Phi_{p}$ are the autoregressive coefficients $\left(y_{t}\right.$ is the degree of dependence on the past); and $\varepsilon_{t}$ is the error term (white noise sequence). The assumption of the model is that $y_{t}$ is only related to historical observations but not related to $y_{t}$ historical series, and $\varepsilon_{t}$ is independent.

The moving average model MA is as follows:

$$
y_{t}=\varepsilon_{t}-\theta_{1} \varepsilon_{t-1}-\theta_{2} \varepsilon_{t-2}-\cdots-\theta_{q} \varepsilon_{t-q},
$$

where $q$ represents the moving average order; $\varepsilon$ means the same as above; and $\theta_{1}, \theta_{2}, \ldots$, and $\theta_{q}$ are moving average coefficients. The moving average model uses the linear combination of random disturbance or prediction error at historical time to express the current observation value.

The calculation method of ARMA is as follows:

$$
\begin{aligned}
y_{t}= & \Phi_{1} y_{t-1}+\Phi_{2} y_{t-2}+\cdots+\Phi_{p} y_{t-p}+\varepsilon_{t} \\
& -\theta_{1} \varepsilon_{t-1}-\theta_{2} \varepsilon_{t-2}-\cdots-\theta_{q} \varepsilon_{t-q} .
\end{aligned}
$$

Assuming that there is a nonstationary time series $u_{t}$, which is converted into a stationary series $u_{t} \sim I(d)$ after $d$ times of difference calculation, then $y_{t}=\Delta^{d} u_{t}=(1-B)^{d} u_{t}$

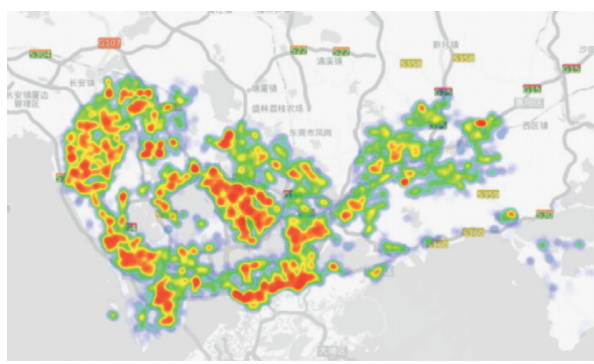

Figure 1: Thermal map of Shenzhen.

and $y_{t}$ are converted into a stationary series and satisfy $y_{t} \sim I(0)$ and the ARMA model can be established. If the processed new sequence satisfies the ARMA model, the original sequence also satisfies the ARIMA model. Therefore, for nonstationary time series, the finite difference can be performed to transform it into a stationary series for prediction. Generally speaking, $d$ does not exceed 2 .

3.2. LSTM Model. Long short-term memory unit is a recurrent neural network (RNN) with memory function. LSTM redefines the memory unit of RNN and uses gate structure to process and predict important events related to long time series [25]. At present, LSTM has been widely used in stock price prediction, text classification, image subtitle, voice modeling, and other fields.

LSTM can not only record the laws in the long sequence but also avoid the disappearance of the gradient and realize the effective use of the long-term sequence information [26]. Figure 2 shows the structure and process of LSTM. The unit for storing historical information in LSTM is composed of memory units in memory blocks and includes three gate structures: input gate, forget gate, and output gate to control the retention and discard of information. Memory block, also known as cell state, is composed of memory line and input-output line.where $h_{t}$ and $h_{t-1}$ represent the current time and the output of the previous neuron; $h_{t-1}$ is the current input; $\sigma$ denotes sigmoid activation function; $W$ is the weight matrix; $f_{t}$ is forgetting gate function; $i_{t}$ is the input gate function; $o_{t}$ is the output gate function; $c_{t}$ and $c_{t-1}$ represent the cell state (memory state) of the current moment; and the previous moment $b_{f}, b_{i}, b_{c}$, and $b_{o}$ indicates the bias condition; and tanh is a hyperbolic tangent function

$$
\begin{aligned}
f_{t} & =\sigma\left(W_{f} \cdot\left(\begin{array}{c}
h_{t-1} \\
x_{t}
\end{array}\right)+b_{f}\right), \\
i_{t} & =\sigma\left(W_{i} \cdot\left(\begin{array}{c}
h_{t-1} \\
x_{t}
\end{array}\right)+b_{i}\right), \\
c_{t} & =f_{t} \otimes c_{t-1}+i_{t} \otimes \tanh W\left(\left(\begin{array}{c}
h_{t-1} \\
x_{t}
\end{array}\right)+b_{c}\right), \\
o_{t} & =\sigma\left(W_{o} \cdot\left(\begin{array}{c}
h_{t-1} \\
x_{t}
\end{array}\right)+b_{o}\right), \\
h_{t} & =o_{t} \otimes \tanh C_{t} .
\end{aligned}
$$




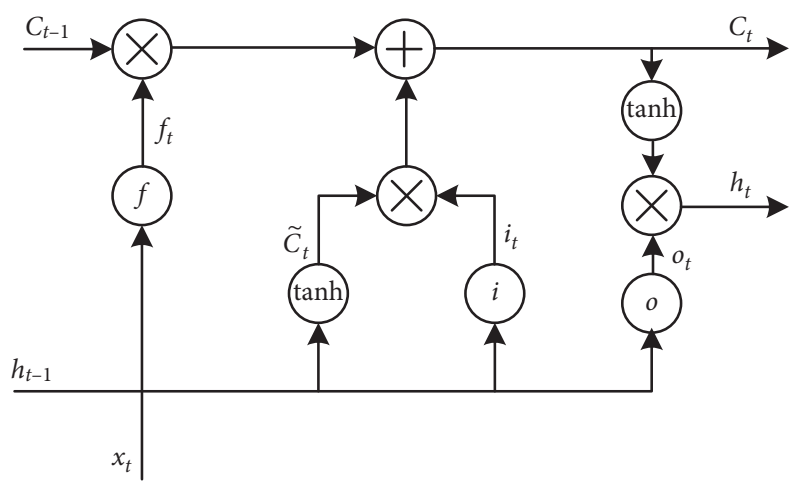

FIGURE 2: LSTM structure diagram.

3.3. ARIMA-LSTM Hybrid Model. Assuming that the time series input of ecological footprint data set is $x_{1}, x_{2}, \ldots, x_{T}$, the ARIMA-LSTM hybrid model has the following forms:

$$
\begin{aligned}
\varphi(B) \nabla^{d} X_{t} & =\theta(B) \varepsilon_{t}, \\
\left(\begin{array}{c}
i_{t} \\
f_{t} \\
o_{t} \\
\widetilde{C}_{t}
\end{array}\right) & =\left(\begin{array}{c}
\sigma \\
\sigma \\
\sigma \\
\tanh
\end{array}\right) W\left(\begin{array}{c}
h_{t-1} \\
x_{t} \\
1
\end{array}\right) .
\end{aligned}
$$

The ARIMA model has a good fitting effect on the linear part of the data, and the LSTM model has a good predictive effect on the nonlinear data. Therefore, this paper proposes an ARIMA-LSTM hybrid model based on the error compensation. In this paper, mean absolute error (MAE) and root mean square error (RMSE) are used to remove abnormal data.

$$
\begin{aligned}
\text { MAE } & =\frac{1}{N} \sum_{i=1}^{N}\left|\left(f_{i}-y_{i}\right)\right|, \\
\text { RMSE } & =\sqrt{\frac{1}{N} \sum_{i=1}^{N}\left(y_{i}-f_{i}\right)^{2} .}
\end{aligned}
$$

The model prediction steps are as follows: the first step uses wavelet denoising method to preprocess the data; the second step establishes an ARIMA prediction model; the third step performs model testing; the fourth step uses ARIMA model prediction; the fifth step establishes LSTM model prediction residual sequence; the sixth step is to add and get the prediction results of the ARIMA-LSTM hybrid model; the seventh step is to perform error analysis. The forecasting flow chart is shown in Figure 3.

3.4. Parallelization of the Weight Calculation Process. The main body of the ARIMA-LSTM model is a complex neural network, which is expanded by a weight matrix. The training process is the update of the weight matrix. Since the calculation of each element of the weight matrix is independent of each other in the same layer, the matrix operation can be parallelized. The basic unit of LSTM is mainly composed of

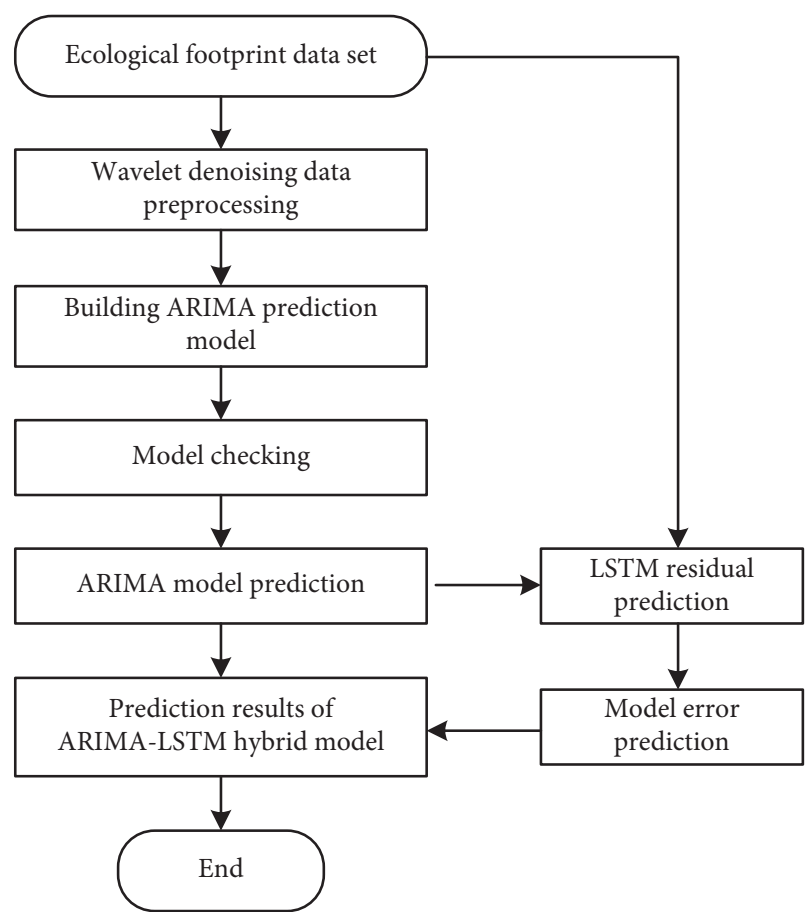

FIgURE 3: Forecast flow chart based on the ARIMA-LSTM model.

three neural network layers whose activation function is sigmoid and one neural network layer whose activation function is Tanh. The computational complexity of the model mainly comes from the gradient calculation in the back-propagation process, but, for each layer of the neural network, this is actually a process of summing the fine-tuned weights calculated for each input.

\section{Effect Verification and Result Discussion}

In order to verify the feasibility of the method proposed in this paper, based on the availability and current situation of data, the experimental data are mainly the current land use data of Shenzhen from 2013 to 2018. The software environment is Python 3.6, and the machine learning framework is Keras. Keras is a neural network API encapsulated in Theano and TensorFlow, which omits the bottom development details and has good modularization and scalability.

4.1. Sensitivity Analysis of the Network. When building the ARIMA-LSTM prediction model, the prediction accuracy is improved by increasing the number of training. Limited by the memory capacity of the hardware, the batch size of this training process is set to 20 , the number of epochs is set to 120 , and the Adam optimizer is used; freezing training is used in the training process, which only updates the parameters of the prediction network in a certain training generation; in this paper, a 3-layer neural network is used to train 60 sample data in each batch. The activation function between the input layer and the hidden layer uses the Tanh function. In the prediction, the number of training is increased from 800 to 2000 , each training is increased by 100 times, and the results are recorded. 


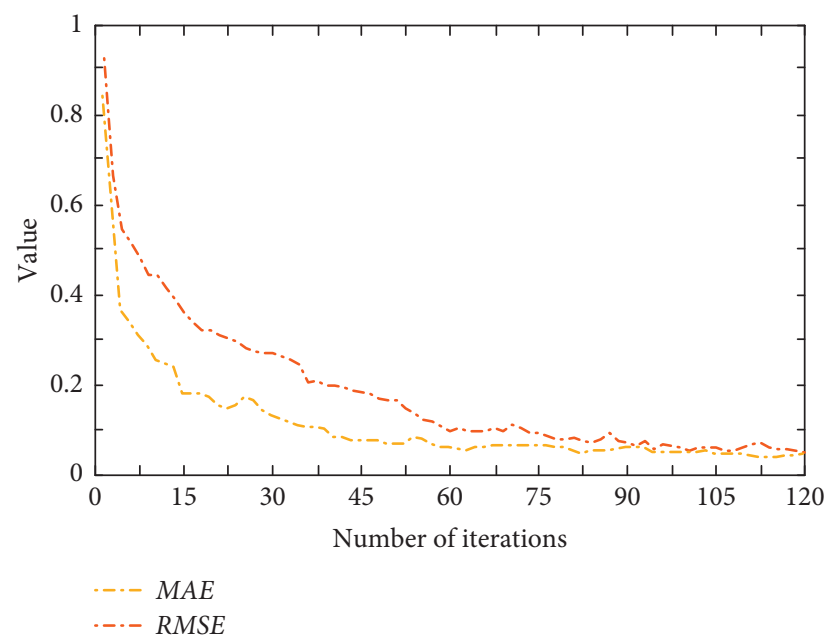

FIGURE 4: ARIMA-LSTM model training process.

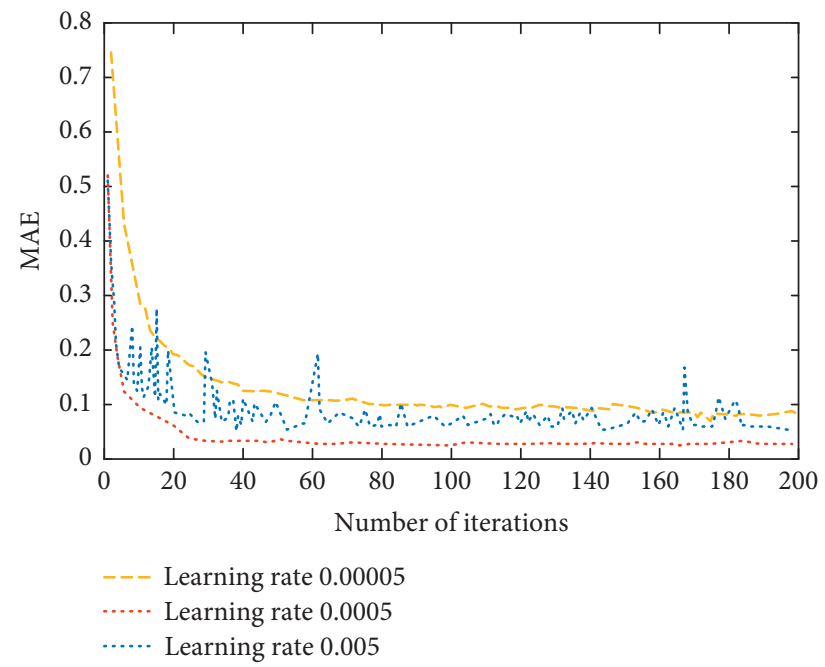

(a)

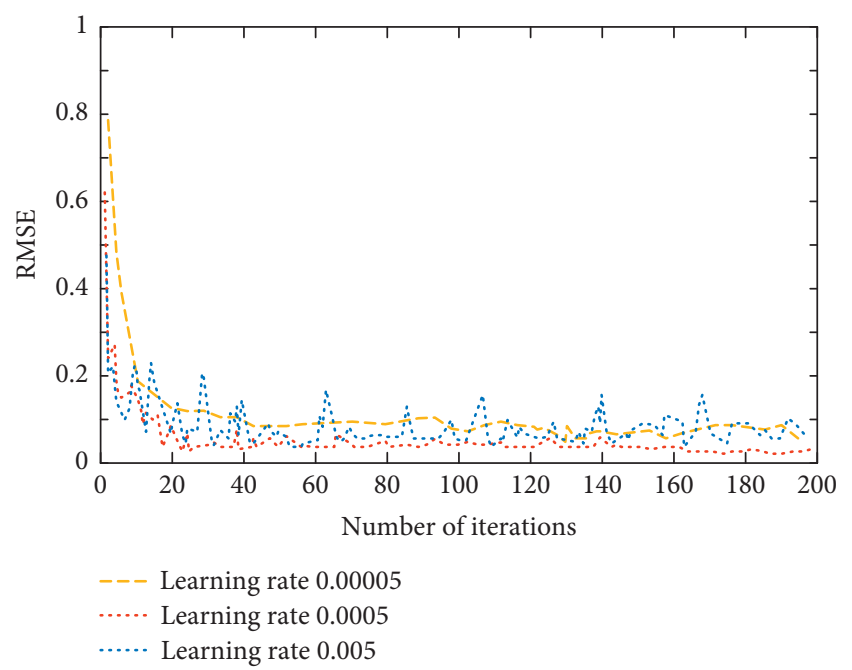

(b)

FIGURE 5: ARIMA-LSTM network performance. (a) MAE. (b) RMSE.

The ARIMA model has a good fitting effect on the linear part of the data, and the LSTM model has a good predictive effect on the nonlinear data. As shown in Figure 4, the ARIMA-LSTM network model optimizes the network performance through 105 epochs, and the average absolute error and root mean square error are optimized in the iterative process.

The rationality of the learning rate parameter setting is very important for the LSTM prediction network model. In this paper, the parameter sensitivity analysis of the set value of the learning rate is carried out. Figure 5 shows the network performance changes under different learning rate values.

As shown in Figure 5, when the network learning rate parameter is set to $0.0005, \mathrm{MAE}$ and RMSE are both optimal, and MAE and RMSE are close to 0 and remain stable during the iteration. The results show that the convergence rate and the convergence limit are better than those with the learning rate parameters of 0.00005 and 0.005 , but the network does not converge effectively when the learning rate is 0.005 .

4.2. Analysis of Urban Evolution. In this paper, each region of Shenzhen is regarded as a whole in the experiment, the per capita ecological footprint of each region from 2013 to 2018 is calculated, and its evolution law in the time axis is shown in Figure 6.

It can be seen from Figure 6 that the per capita ecological footprint of Shenzhen shows a downward trend and is greatly affected by the ecological footprint of cultivated land, from $2.781 \mathrm{hm}^{2} /$ person in 2013 to $2.292 \mathrm{hm}^{2} /$ person in 2018 . The overall trend of per capita ecological footprint is basically the same as that of buildings. The main reason is that the study area, as the best comprehensive condition area in Guangdong Province, has developed urban construction and a high ecological footprint. The per capita ecological 


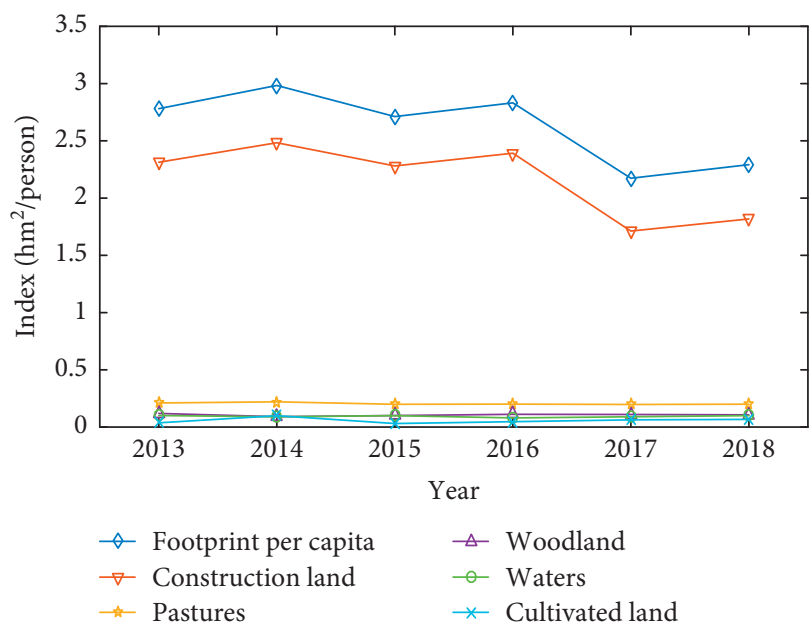

FIgURE 6: Temporal evolution of per capita ecological footprint.

footprint showed an obvious turning point in 2016, mainly due to the low pork price and the low price of beef and mutton during this period, which led to a sharp decline in the enthusiasm of farmers and a significant drop in meat production.

According to the ecological carrying capacity model, the per capita ecological carrying capacity from 2013 to 2018 (deduct $12 \%$ for biodiversity maintenance) is calculated and then subtracted it from the per capita ecological footprint value to calculate the per capita ecological loss, and the specific evolution is shown in Figures 7 and 8.

It can be seen from Figures 7 and 8 that the per capita carrying capacity of Shenzhen from 2013 to 2018 has shown a relatively stable and declining trend, while the per capita ecological loss is mainly manifested under deficit, but the overall trend is positive. The stability of per capita carrying capacity is mainly due to the implementation of the strictest cultivated land protection system in China. Although the cultivated land area is decreasing year by year, the decreasing speed is gradually slowing down.

4.3. Analysis of Sustainable Development. In order to study the development prospect of Shenzhen, this paper further studies and analyzes its sustainability, as shown in Figure 9.

It can be seen from Figure 9 that the economic development capacity index of Shenzhen has increased significantly over the years. However, the diversity of ecological footprint has increased slightly, which indicates that the capacity of economic development has been continuously improved. Further analysis shows that the improvement of economic development capacity is based on the substantial increase of ecological footprint.

At the same time, this paper also carries on the corresponding ecological analysis to the tourism development of Shenzhen, and Figure 10 is the time series chart of per capita tourism ecological surplus.

It can be seen from Figure 10 that the local sustainable development is mainly manifested in the per capita tourism ecological surplus, which has a gradually decreasing development trend over time. It can be explained that the

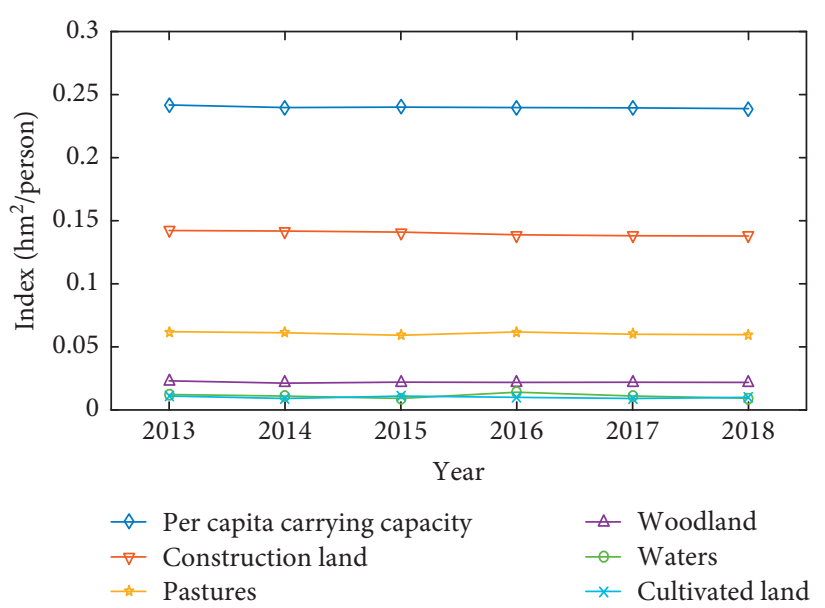

FIgURE 7: Time evolution of per capita carrying capacity.

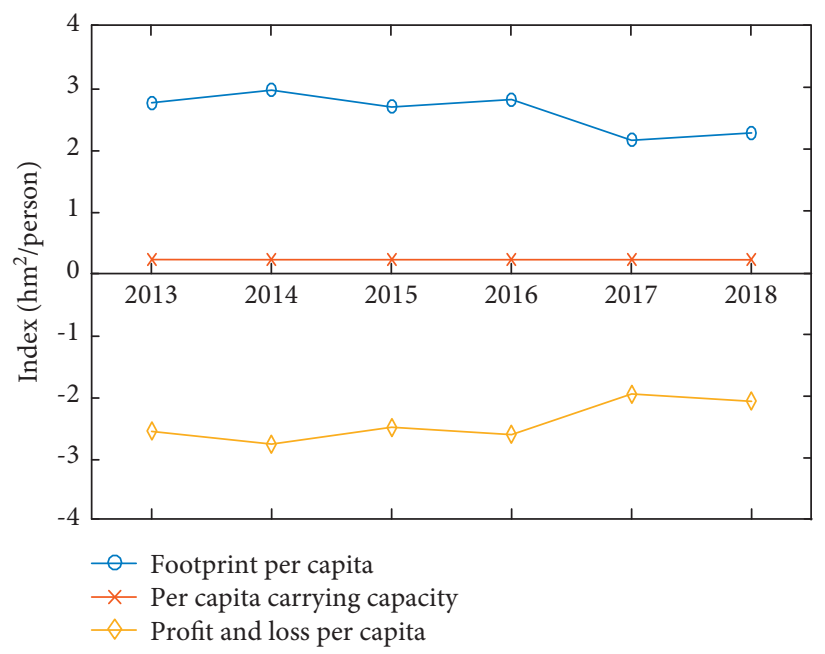

FIgURE 8: Evolution of three ecological indexes.

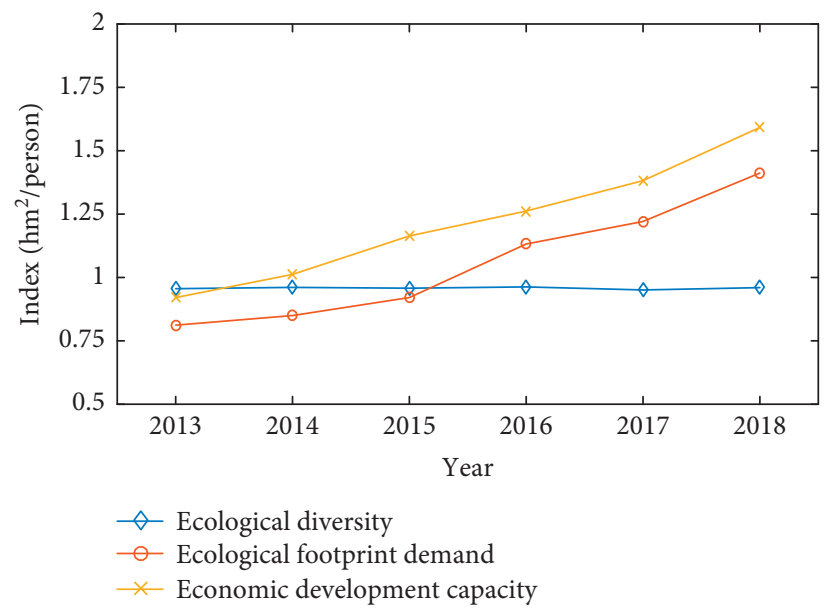

FIGURE 9: Sustainable development in Shenzhen.

excessive increase of the tourism population will affect the local tourism ecological environment, thus making the per capita tourism ecological surplus gradually close to zero or 


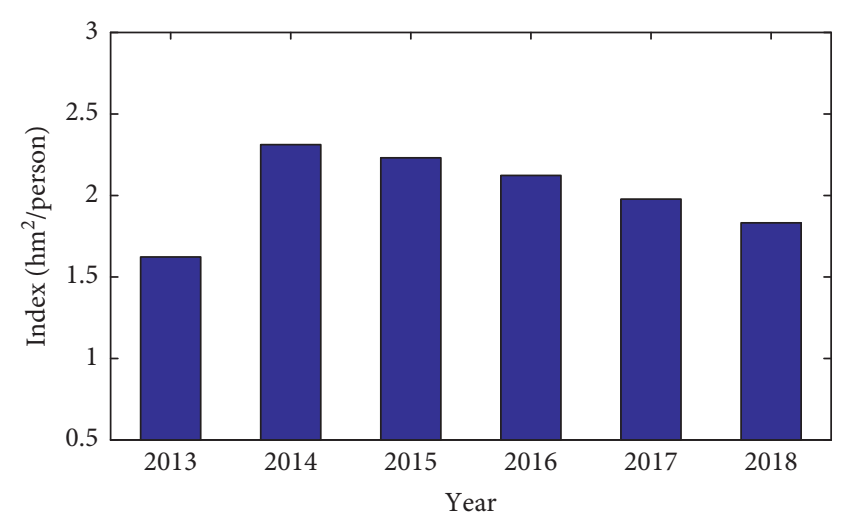

Figure 10: Time series chart of per capita tourism ecological surplus.

even lower than zero. According to this trend, if the number of tourists is not controlled, a tourism ecological deficit will occur in the near future, which will damage the local tourism ecological environment.

\section{Conclusion}

Aiming at the problem of large errors in the current regional ecological evaluation and analysis methods, this paper realizes the prediction of the per capita ecological carrying capacity of Shenzhen based on the energy analysis method and artificial intelligence technology. This paper adopts the energy analysis method to improve the ecological footprint model, realizes a more comprehensive and reliable modeling analysis of the regional ecological footprint data model, and provides complete data support for the fusion network prediction model; at the same time, in order to further improve the network prediction ability, the ARIMA and LSTM networks are integrated to comprehensively analyze the linear and nonlinear data at the same time, so as to ensure the optimal performance of the prediction network. Through the analysis of the evolution law and sustainable development capacity of the actual collected data set in Shenzhen, it can be seen that the overall development trend of Shenzhen's economy is favorable, but attention should be paid to the problem of tourism ecological deficit.

\section{Data Availability}

The data used to support the findings of this study are included within the article.

\section{Conflicts of Interest}

The author declares that there are no conflicts of interest regarding the publication of this paper.

\section{Acknowledgments}

This work was supported by the Culture and Tourism Department of Shaanxi Provincial Government (Nos. SXLYKT201600 and H201737) and Education Department of Shaanxi Provincial Government (No. 18JK0632).

\section{References}

[1] M. Tang, "A novel remote sensing evaluation method of ecological environment carrying capacity of elderly community," Fresenius Environmental Bulletin, vol. 30, no. 3, pp. 2871-2878, 2021.

[2] M. Zhao and S. S. Hao, "Prediction model of ecological environment carrying capacity in the waters of hainan island," Journal of Coastal Research, vol. 93, no. 1, pp. 436-442, 2019.

[3] W. Dong, "Research on energy saving planning of urban high-density green space based on digital remote sensing images," Fresenius Environmental Bulletin, vol. 29, no. 10, pp. 9075-9081, 2020.

[4] K. Zhang and D. F. Wang, "Assessment method of environmental carrying capacity of green ecotourism based on tourists' perception and experience," Fresenius Environmental Bulletin, vol. 29, no. 9A, pp. 8584-8591, 2020.

[5] J. Han, "Carrying capacity of low carbon tourism environment in coastal areas from the perspective of ecological efficiency," Journal of Coastal Research, vol. 83, no. 1, pp. 199-203, 2018.

[6] Y. Wang, RY. Shi, and CL. Qiao, "Evaluation of water resources carrying capacity based on fuzzy comprehensive evaluation method in northern slope economic belt of tianshan mountains," Bulletin of Soil and Water Conservation, vol. 38, no. 5, pp. 206-212, 2018.

[7] ZF. Wang, YJ. Wang, L. Wang, T. Zhang, and Z. Tang, "Research on the comprehensive evaluation system of ecogeological environmental carrying capacity based on the analytic hierarchy process," Cluster Computing-The Journal of Networks Software Tools and Applications, vol. 22, no. 1, pp. 5347-5356, 2019.

[8] P. Mao, AW. Lin, Q. Yang, and H. J. Zhu, "Evaluation of regional ecological carrying capacity of urban agglomeration in the middle reaches of the Yangtze River Based on state space method," Mapping and spatial geographic information, vol. 40, no. 3, pp. 37-41, 2017.

[9] Y. Wang, B. Peng, G. Wei, and E Elahi, "Comprehensive evaluation and spatial difference analysis of regional ecological carrying capacity: a case study of the yangtze river urban agglomeration," International Journal of Environmental Research and Public Health, vol. 16, no. 18, pp. 1-16, 2019.

[10] BW. Wang and Y. Feng, "Swot analysis on tourist ecofootprint and ecological carrying capacity in poverty-stricken areas," ENVIRONMENTAL ENGINEERING AND MANAGEMENT JOURNAL, vol. 19, no. 9, pp. 1481-1489, 2020.

[11] X. Yang, "Study on the coordinated development of regional economic, tourism and ecology coupling: taking henan Province as an example," Fresenius Environmental Bulletin, vol. 30, no. 1, pp. 210-215, 2021.

[12] C. S. Jin, R. J. Deng, Y. X. Liu, B. Ren, and H. Xiao, "Spatiotemporal analysis and prediction of water resources ecological footprint in Yangtze River economic belt," Journal of water resources and water engineering, vol. 29, no. 4, pp. 62-69, 2018.

[13] J. Huertas, F. Farinha, M. J. Oliveira, and R. L. SilvaEMJ, "Comparison of artificial intelligence algorithms to estimate sustainability indicators," Sustainable Cities and Society, vol. 63, no. 1, pp. 1-13, 2020.

[14] Y. Liu, T. Wang, and GH. Fang, "Integrated prediction and evaluation of future urban water ecological sustainability from the perspective of water ecological footprint: a case study of jinan, China," Fresenius Environmental Bulletin, vol. 27, no. 10, pp. 6469-6477, 2018. 
[15] L. Liu and Y. Lei, "An accurate ecological footprint analysis and prediction for Beijing based on SVM model," Ecological Informatics, vol. 44, no. 1, pp. 33-42, 2018.

[16] S. Jiang, C. Lu, S. Zhang et al., "Prediction of ecological pressure on resource-based cities based on an RBF neural network optimized by an improved $\mathrm{ABC}$ algorithm," IEEE Access, vol. 7, no. 1, pp. 47423-47436, 2019.

[17] J. Yang, B. Zheng, and Z. Chen, "Optimization of tourism information analysis system based on big data algorithm," Complexity, vol. 2020, no. 1, pp. 1-11, 2020.

[18] HuangZQ, "Partial least squares regression analysis to factor of influence for ecological footprint," Cluster Computing, vol. 22 , no. 3, pp. 6425-6433, 2019.

[19] S. L. Liu, J. L. Zhu, JW. Xu, X. Wu, S. Zhao, and X. Y. Hou, "Effect of urbanization on the ecological footprint and their interactive coupling relationship," Acta Ecologica Sinica, vol. 38, no. 24, pp. 8888-8900, 2018.

[20] RM. Gogonea, SI. Ghita, and AS. Saseanu, "Biocapacitypremise of sustainable development in the European space," Sustainability, vol. 12, no. 3, pp. 1-26, 2020.

[21] HJ. Bao, CC. Wang, L. Han, S. Wu, and Y. Liu, "Resources and environmental pressure, carrying capacity, and governance: a case study of yangtze river economic belt," Sustainability, vol. 12, no. 4, pp. 1-18, 2020.

[22] Y. Su, W. Gao, D. Guan, and W. Su, "Dynamic assessment and forecast of urban water ecological footprint based on exponential smoothing analysis," Journal of Cleaner Production, vol. 195, no. 10, pp. 354-364, 2018.

[23] LP. Wu and J. Zhang, "Dynamic distribution and prediction of inbound tourists in China based on ARIMA model with dummy variables," Journal of Tourism, vol. 30, no. 11, pp. 74-86, 2015.

[24] ZH. Wang, L. Yang, JH. Yin, and B. Zhang, "Assessment and prediction of environmental sustainability in China based on a modified ecological footprint model," Resources, Conservation and Recycling, vol. 132, no. 1, pp. 301-313, 2017.

[25] L. Yang, YX. Wu, and J. L. Wang, "Review of recurrent neural networks," Computer Applications, vol. 8, no. 2, pp. 1-6, 2018.

[26] QY. He, HP. Li, and YX. Ou, "Coupling coordination analysis and simulation prediction of urbanization and ecological environment of urban agglomeration in the middle reaches of the Yangtze River," Ecological science, vol. 39, no. 02, pp. 182-190, 2020. 